\title{
COMPARATIVE CLINICAL STUDY OF NON-MESH VERSUS MESH REPAIR IN PRIMARY INGUINAL HERNIA
}

\section{Surgery}

\section{Dr. Palash Saha}

\section{Dr. Ved Rajan} Arya*

\section{Dr. Debarshi Jana}

Assistant Professor, Department of Surgery, Gouri Debi Medical College, Rajbandh, Durgapur, West Bengal.

Associate Professor, Department of Surgery, MGM Medical College, Kishanganj, Bihar.

*Corresponding Author

IPGMER and SSKM Hospital, Kolkata, West Bengal.

\section{ABSTRACT}

BACKGROUND: The inguinal regions are one of the natural weak areas in the abdominal wall and are the most common site of herniation. Inguinal hernia repair is one of the most frequently performed operations. AIMS: The aim of this study is to compare open mesh and non-mesh suture repair of primary inguinal hernias with respect to clinical outcome which include postoperative pain, complications, time of return to work and recurrence. MATIERIALAND METHODS: This prospective study of 102 cases of primary inguinal hernia repair was carried out at M.G.M. Medical College \& L.S.K. Hospital, Kishanganj with the aim of comparing open mesh and non-mesh suture repair with respect to clinical outcome which included postoperative pain, complications time of return to work and recurrence. RESULT: In our study there were no specific criteria for allocation of the method of repair and of 102 patients 52 patients randomly underwent non-mesh repair and 50 patients underwent mesh repair. CONCLUSION: The use of prosthetic mesh allows tension-free repair of inguinal hernia and in theory better results. Our study proves the superiority of this method over non-mesh repair in the long term with regard to hernia recurrence; in addition, there were less complications and postoperative pain.

\section{KEYWORDS}

Primary Inguinal Hernia, Open Mesh, Herniation, Mesh Repair

\section{INTRODUCTION}

A hernia is defined as a protrusion of a viscus through an opening in the wall of the cavity in which it is contained. Hernias of the abdominal wall occur only in areas where aponeurosis and fascia are devoid of the protecting support of striated muscle. Without a counteracting force, the bare aponeurotic areas are subjected to the ravages of intraabdominal pressure and give way if they deteriorate or contain anatomical irregularities.

The incidence of inguinal hernia in adults varies between $10 \%$ to $15 \%$. The male to female ratio is $12: 11$. Hernias are present in more than $45 \%$ of males at 75 years of age and older.

Inguinal hernia repair is one of the most frequently performed operations. Failure of inguinal hernia repair leads to increased patient discomfort and re-operations results in a considerable burden on the patient. Chances of hernia recurrence after open surgery ranges from $1 \%$ to $7 \%$ for an indirect hernia and from $4 \%$ to $10 \%$ for a direct hernia . Though there are various reasons for the failure of repair, the method of repair plays a vital role in the success of inguinal hernia repair. Until recently the standard procedure has been open musculo-aponeurotic repair using sutures under tension to close the defect but 'tension- free' repair using prosthetic mesh is becoming increasingly common in many countries. Use of Lichtenstein mesh repair increased from $33 \%$ in January 1998 to $62 \%$ in June $2000^{2}$. No consensus has yet been reached about the surgical approach to inguinal hernia repair that gives best clinical result ${ }^{3}$. The optimal method for inguinal hernia repair is yet to be determined ${ }^{3}$

The aim of this study is to compare open mesh and non-mesh suture repair of primary inguinal hernias with respect to clinical outcome which include postoperative pain, complications, time of return to work and recurrence.

\section{MATERIALS AND METHODS}

This study had been conducted on patients admitted for primary inguinal hernia who were either repaired by non-mesh suture method using $2 / 0$ prolene suture or by open polypropylene (Prolene) prosthetic mesh. The study was done at M. G. M. Medical College and Lions Seva Kendra Hospital, Kisanganj. Prospective study was carried out on cases performed between dec 2010 to August 2012 which included 102 patients who underwent primary inguinal hernia repair. Of the 102 patients, 52 patients underwent non-mesh repair whereas 50 patients underwent open mesh repair.

All patients older than 18 years scheduled for repair of a primary inguinal hernia were randomised to either non-mesh or mesh repair.
Patients were enrolled only once. Age, Sex, intermittent high abdominal pressure i.e., persistent cough, constipation, prostatism and factors that may interfere with wound healing i.e., diabetics, use of steroids, smoking were noted. All patients were thoroughly examined, the type of inguinal hernia diagnosed clinically was noted and basic investigations were done.

One hour before operation a single dose of intravenous Cefotaxime was administered. Patients underwent operation under either spinal anaesthesia with Bupivacaine or local anaesthesia consisting of $0.5 \%$ lignocaine and $0.25 \%$ bupivacaine administered by local-layer infiltration. All patients were monitored by pulse oximeter.

\section{RESULT AND DISCUSSION}

In our prospective study of 102 cases who underwent primary hernia repair, 100 were male patients and 2 were female patients. $60 \%$ of the cases were above the age of 50 years and $60 \%$ were right sided.

18 cases randomly received local anaesthesia whereas 84 cases received spinal anaesthesia. No cases of urinary retention were found in those who received local anaesthesia but 26 cases (30\%) of those who received spinal anaesthesia developed urinary retention in the postoperative period and had to be catheterised. Early ambulation soon after operation was also possible in the cases who received local anaesthesia.

AU Kark $\mathrm{AE}^{4}$ in 1998 in the study of 3175 primary inguinal hernia repairs using polypropylene mesh under local anaesthesia had no cases of urinary retention. All open hernia techniques lend themselves ideally to local anaesthesia. Open mesh repair under local anaesthesia is an effective day care technique particularly in the elderly and medically unfit.

Median duration of Surgery for non-mesh repair was 40 minutes (range 30 - 90 minutes) and for mesh repair was 45 minutes (range $40-$ 90 minutes). AU Janu PG et $\mathrm{l}^{5}$ reported that operative time for mesh repair (111+/-2 minutes) was longer than for Bassini or MC Vay (91+/2 and $98+/-2$ minutes).

In our study there were no specific criteria for allocation of the method of repair and of 102 patients 52 patients randomly underwent nonmesh repair and 50 patients underwent mesh repair.

Both groups experienced pain in the postoperative period. The intensity of pain at 24 hours, 48 hours and 7 days after surgery was greater in the non-mesh repair group. $60 \%$ of the patient experienced persisting pain and had to be given oral Diclofenac $50 \mathrm{mg}$ daily for 5 
days. Intensity of pain was less in the mesh repair group and $20 \%$ of the patients experienced persisting pain. As reported by AU EU Hernia trialists ${ }^{6}$ collaboration in March 2002; mesh repair appeared to reduce the chance of persisting pain rather than increasing it. However AU Callesen T et al ${ }^{7}$, had reported in 1999 that choice of surgical technique for open repair of a primary inguinal hernia has no influence on post operative pain.

Neuropraxia and hypesthesia have been reported in $15 \%$ to $20 \%$ of patients following open hernia repair, whereas chronic pain was seen in $5 \%$ cases.

In our study median hospital stay was 5 days (range $2-10$ days) for non-mesh repair group and 3 days (range $1-5$ days) for mesh repair group. AU Pavlidis TE et $\mathrm{al}^{8}$ reported in 2002 that hospital stay in nonmesh repair cases was 4.2 days as compared to 1.8 days in mesh repair cases in primary inguinal hernia.

Time off work was 21 days (range 7-45 days) for non-mesh repair group and 19 days (range 7-30 days) for mesh repair group. It was found perhaps not surprisingly that manual workers took slightly longer (median of 30 days) than did desk workers or retirees (median of 20 days) to return to normal routine. AU Pavlidis TE et al ${ }^{8}$ reported in their study that return to work for non-mesh repair cases was 17.2 days as compared to 7.3 days in mesh repair cases in primary inguinal hernia.

Post operative wound infection is one of the most serious complication of repair of groin hernias. In our study 1 case (2\%) of mesh repair group had a localized stitch abscess which subsided following intravenous Cefotaxime for 5 days. In the non-mesh repair group 3 cases $(5.7 \%)$ had acute cellulites around the wound with pus discharge from the open wound. The phase settled down following intravenous Cefotaxime and regular dressing and the wound healed. All these patients who had wound infections were of the elderly age group. It has been reported that older patients specifically over 70 years of age show a 3.2 fold higher incidence of wound infections. AU Janu PG et $\mathrm{al}^{5}$ in his study of 892 patients reported $1 \%$ infection in those who had mesh repair and $1.8 \%$ in those who had non-mesh repair.

Hematoma occurred in 2 cases $(3.8 \%)$ of non-mesh group while subsided after aspiration whereas it was not noted in any case of mesh repair group. Seroma was noted in 4 cases $(7.7 \%)$ of non-mesh repair group.

AU Scott NW et al ${ }^{9}$ reported in 2002 that there were no clear differences between mesh and non-mesh groups for hematomas, seromas or wound infections.

During the 2 years follow up 1 case $(1.9 \%)$ recurrence was found in the non-mesh repair group and none in the mesh repair group. The case which recurred was male and more than 70 years of age. The recurrence was noted during the first year of postoperative period. The case didn't had any postoperative complication. Though $50 \%$ of recurrences are caused by infection, in our study infection was not associated with recurrence.

The case was non-smoker and didn't had any co-morbid conditions like chronic cough or prostatism. A higher percentage of smoker develops recurrences after repair as systemic protease/antiprotease imbalance in them leads to fascial degeneration and interference with normal wound healing. Abramson and colleagues showed that chronic cough was not associated with recurrence. As such prostatic hypertrophy is of no significance in the development of a hernia or recurrence after repair.

The approximation of tissues under tension is a cardinal factor in the failure of a hernia repair. Tissues sutured under tension tend to pull apart but are prevented from doing so by the sutures; however, the tissue pulling on the sutures create an area of ischemic pressure necrosis where the suture meets the tissue. This process of ischemic necrosis progresses until there is no longer any tension, which usually occurs when the tissues have returned to their previous unsutured position and the hernia recurs through the resultant gap. In more extreme cases in which the tension is greater than the strength of the tissues, the sutures simply tear the tissues and the hernia recurs.

Danielson et $\mathrm{a}^{10}$ compared the Shouldice technique and Lichtenstein tension free mesh technique in 178 patients operated upon by trainees.
Duration of operation and complication rates was similar. Mesh repair resulted in less days of sick leave $(18$ versus 23 days, $p<0.05)$ and fewer recurrences (nil versus 9, $\mathrm{p}<0.01$ ). He concluded that for surgeons in training the Lichtenstein open mesh technique is a better method of inguinal hernia repair than the Shouldice technique.

"Comparison of non-mesh (Desarda) and mesh (Lichtenstein) methods for inguinal hernia repair among black African patients and the best result shows in mesh repair hernia. Study was done for 150 patient and in the study population 70 patient were subjected to nonmesh (Desarda) and the rest underwent mesh (Lichtenstein) methods.

AU Scott NW et al ${ }^{9}$ reviewed 21 trials comparing open mesh with open non-mesh repair in primary inguinal hernia. Open mesh methods on average took 7-10 minutes less to perform than Shouldice procedures but took 1-4 minutes longer than other non-mesh methods. Those in the mesh groups had a shorter length of hospital stay and quicker return to usual activities. They concluded that the use of open mesh repair is associated with a reduction in the risk of recurrence between $50 \%$ and $75 \%$

AU Janu PG, Seller KD et al ${ }^{5}$ did a study to compare mesh versus nonmesh inguinal herniorrhaphy. From 1985 to 1994892 patients underwent primary repair for inguinal hernia at the Veteran's Administration Hospital at Memphis, TN. Mesh patients developed four wound infections $(1.0 \%)$ none requiring mesh removal versus nine infections $(1.8 \%)$ in non-mesh repair. One mesh patient $(0.3 \%)$ developed recurrence compared with $16(3.5 \%)$ with open anterior repair $(\mathrm{p}<0.01)$.

In a systematic review of randomized controlled trials carried out by $\mathrm{AU}$ collaboration $\mathrm{EH}^{11}$, in was reported that return to usual activities was quicker in the mesh group. There were fewer recurrences in the mesh group; $21(1.4 \%)$ of 1513 versus $72(4.4 \%)$ of $1634(p<0.001)$

AU Bay - Nielsen ${ }^{2}$ did a prospective study of 26,304 groin hernia repair in Denmark between Jan 1, 1998 to Jan 30, 2000 and reported that reoperation rates 30 months after anterior mesh repair and laparoscopic repair were significantly lower than after sutured posterior wall repair in primary inguinal hernia $(2.2 \%$ and $2.6 \%$ vs $4.4 \% ; \mathrm{p}<0.0001$ ). Use of Lichtenstein mesh repair increased from $33 \%$ in January 1998 to $62 \%$ in June 2000 whereas use of laparoscopic repair remained constant at about $5 \%$. Reoperation rates were $2.8 \%$ in the first 15 months and $1.6 \%$ in the second.

A retrospective comparison of 182 onlay mesh repairs and 170 Bassini repairs carried out by surgeons in training found five recurrences $(3.3 \%)$ in the mesh repair group and $13(11.5 \%)$ in the Bassini group.

\section{CONCLUSION}

Inguinal hernia repair performed by suturing and displacement of anatomic structures may lead to excessive tension on the suture line and surrounding tissue. The use of prosthetic mesh allows tension-free repair of inguinal hernia and in theory better results. Our study proves the superiority of this method over non-mesh repair in the long term with regard to hernia recurrence; in addition, there were less complications and postoperative pain. Duration of hospital stay was less and return to work was earlier following mesh repair. Therefore, mesh repair is the method of choice for primary inguinal hernia repair.

\section{REFERENCES}

1. Scott DJ, Jones DB (2000). Hernias and abdominal wall defects. In JA Norton et al, eds., Surgery: Basic Science and clinical Evidence, PP 803-810 New York: Springer.

2. AUBay- Nielson M. Kehlet H. Strand L. Malmstrom J. Anderson FH. Wara P. Juul p. Callesen T. Danish Hernia Database Collaboration T1 Quality assessment of 26, 304 herniorhapheis in Denmark: a prospective nation wide study SO Lancet. 358 (9288): 11 24-8,2001 October 6 .

3. AV Vrijland WW. Van den Tol MP. Luijendijk RW. Hop WC. Busschbach JJ. De large DC. Van Geldane D. Rottier AB. Vegt PA. Ijzermans JN. Jeekel J. Randomised clinical trial of non mesh versus mesh repair of primary inguinal hernia; British Journal of surgery.89(3): 293-7,2002 March

4. AU Kark AE, Kurzer MN, Belsham PA; Three thousand one hundred seventy five primary inguinal hernia repairs: advantages of ambulatory open mesh repair using local primary inguinal hernia repairs: advantages of ambulatory open mesh repair using local anaesthesia; Journa

5. AU Janu PG, Sellers KD, Mangiante EC; Mesh inguinal herniorraphy: a ten-year review. American Surgeon. 63(12):1065-9, 1997 December

6. AU EU Hernia Trialists Collaboration; Repair of groin hernia with synthetic mesh: meta-analysis of randomised controlled trials; Annals of Surgery: 235(3): 322-32, 2002 March.

7. AU Callesen T, Bech K, Anderson J, Nielsen R, Roikjaer O, Kehlet H; Pain after primary inguinal herniorraphy: influence of surgical technique; journal of the American college of Surgeons. 188(4): 355-9, 1999 April.

8. AU Pavlidis TE. Atmatzidis KS. Lazaridis CN. Papaziogas BT, Makris JG. Papaziogas 
TB; Comparison between modern mesh and conventional non- mesh methods of inguinal hernia repair; Minerva Chirurgica. 57(1): 7-12,2002 February.

9. AU Scott NW, McCormack K, Graham P, GOPM, Ross SJ, Grant AM: open Mesh versus non- mesh for repair of femoral and inguinal hernias; Cochrane Database of Systematic Reviews.(4): CD002197,2002

10. AU Danielsson P, Isacson S, Hansen MV: Randomised study of Lichtenstein compared with Shouldice inguinal hernia repair by surgeons in training; European Journal of Surgery, 165 (1): 49-53, 1999 January

11. AU Collaborations EH: Mesh compared with non- mesh methods of open groin hernia repair: systematic review of randomised controlled trials; British Journal of Surgery. 87(7): 854-9, 2000 July. 